Original article

\title{
Unmet needs in patients with brief psychotic disorders: Too ill for clinical high risk services and not ill enough for first episode services
}

\author{
Amedeo Minichino ${ }^{\mathrm{a}, \mathrm{b}}$, Grazia Rutigliano $^{\mathrm{a}}$, Sergio Merlino ${ }^{\mathrm{a}}$, Cathy Davies ${ }^{\mathrm{a}}$, \\ Dominic Oliver ${ }^{\mathrm{a}}$, Andrea De Micheli ${ }^{\mathrm{a}}$, Rashmi Patel ${ }^{\mathrm{c}}$, Philip McGuire ${ }^{\mathrm{c}}$, \\ Paolo Fusar-Poli ${ }^{\mathrm{a}, \mathrm{d}, \mathrm{e}, *}$ \\ ${ }^{a}$ Early Psychosis: Interventions E Clinical-detection (EPIC) lab, Department of Psychosis Studies, King's College London, Institute of Psychiatry Psychology and \\ Neuroscience, London, United Kingdom \\ ${ }^{\mathrm{b}}$ Department of Psychiatry, University of Oxford, Oxford, United Kingdom \\ ${ }^{\mathrm{c}}$ Department of Psychosis Studies, King's College London, Institute of Psychiatry Psychology and Neuroscience, London, United Kingdom \\ d OASIS Service, South London and the Maudsley NHS Foundation Trust, London, United Kingdom \\ e Department of Brain and Behavioral Sciences, University of Pavia, Pavia, Italy
}

\section{A R T I C L E I N F O}

\section{Article history:}

Received 20 September 2018

Received in revised form 12 December 2018

Accepted 13 December 2018

Available online 15 January 2019

\section{Keywords:}

Psychosis

Schizophrenia

Risk

Prevention

ATPDs

\begin{abstract}
A B S T R A C T
Background: Patients with acute and transient psychotic disorders (ATPDs) are by definition remitting, but have a high risk of developing persistent psychoses, resembling a subgroup of individuals at Clinical High Risk for Psychosis (CHR-P). Their pathways to care, treatment offered and long-term clinical outcomes beyond risk to psychosis are unexplored. We conducted an electronic health record-based retrospective cohort study including patients with ATPDs within the SLaM NHS Trust and followed-up to 8 years.

Methods: A total of 2561 ATPDs were included in the study. A minority were detected (8\%) and treated (18\%) by Early Intervention services (EIS) and none by CHR-P services. Patients were offered a clinical follow-up of $350.40 \pm 589.90$ days. The cumulative incidence of discharges was $40 \%$ at 3 months, $60 \%$ at 1 year, $69 \%$ at 2 years, $77 \%$ at 4 years, and $82 \%$ at 8 years. Treatment was heterogeneous: the majority of patients received antipsychotics (up to $52 \%$ ), only a tiny minority psychotherapy (up to $8 \%$ ).

Results: Over follow-up, $32.88 \%$ and $28.54 \%$ of ATPDS received at least one mental health hospitalization or one compulsory hospital admission under the Mental Health Act, respectively. The mean number of days spent in psychiatric hospital was $66.39 \pm 239.44$ days.

Conclusions: The majority of ATPDs are not detected/treated by EIS or CHR-P services, receive heterogeneous treatments and short-term clinical follow-up. ATPDs have a high risk of developing severe clinical outcomes beyond persistent psychotic disorders and unmet clinical needs that are not targeted by current mental health services.
\end{abstract}

(c) 2018 Elsevier Masson SAS. All rights reserved.

\section{Introduction}

Short-lived psychotic episodes are traditionally classified as Brief Psychotic Disorders (BPD, in DSM-V) and Acute and Transient Psychotic Disorders (ATPDs in ICD-10), respectively [1]. Recently, patients presenting with short-lived psychotic episodes have been included in the Brief Limited Intermittent Psychotic Symptoms (BLIPS) or Brief and Intermittent Psychotic

\footnotetext{
* Corresponding author at: Department of Psychosis Studies, Institute of Psychiatry, Psychology \& Neuroscience, PO63, 5th Floor, 16 De Crespigny Park, SE5 8AF, London, UK.

E-mail address: paolo.fusar-poli@kcl.ac.uk (P. Fusar-Poli).
}

Symptoms (BIPS) subgroups of the Clinical High Risk state for Psychosis (CHR-P) [2,3]. There is a diagnostic overlap ( 70\%) between BLIPS and ATPDs [4]. Whichever the designation used, patients with short-lived psychotic episodes share: (i) psychotic symptoms with a brief duration from 7 days (BLIPS) to 3 months (ATPDs) [4], and (ii) a very high risk of developing persistent psychotic disorders. This risk accumulates to 0.51 [0.41-0.61] at 3-years [5]. Specifically, one-fourth of patients with an initial ATPDs diagnosis would develop schizophrenia-spectrum psychoses and up to one-third affective psychoses [6]. However, although about one in two ATPDs patients will develop persistent psychotic disorders at follow-up, their risk is lower than the risk observed in patients with an initial first-episode of schizophrenia who remitted from their symptoms [5]. 
There is limited knowledge with respect to ATPDs': (i) pathways to care (i.e., which clinical services detect, treat and follow-up these patients),(ii) treatment offered and (iii) long-term outcomes beyond the risk of developing persistent psychotic disorders in clinical practice. The paucity of research in this area may reflect the fact that these patients are by definition remitted at the time of their index diagnosis and therefore their perceived need of care may be low, an assumption largely unverified. For example, in the UK the National Health Service (NHS) Mental Health Trusts assume that Early Intervention Services (EIS) for psychosis and CHR-P services are the deputy services to take care of these individuals, under the ATPDs or BLIPS/BIPS designations, respectively [7]. Yet, it is undetermined how many ATPDs are actually detected and treated by EIS or CHR-P services in the real-world clinical routine. Furthermore, under current guidelines, patients presenting with a short-lived psychotic episode may either be recommended conventional antipsychotic treatment (if diagnosed with ATPDs and according to the current EIS guidelines) [8] or be contraindicated antipsychotic treatment and receive psychological therapies (if diagnosed with BLIPS by CHR-P services) [8]. Yet, the actual type of care which is offered to these patients is unknown. For example, it is undetermined how many ATPDs patients would actually undergo antipsychotic treatment or whether they would undergo any other types of treatment such as psychotherapy or clinical monitoring. Further, mental health outcomes other than the risk to develop a persistent psychotic disorder, such as number of admissions in mental health hospitals, days spent in hospital, and use of Mental Health Act (MHA) assessment in this population are unknown.

Overall, the lack of knowledge about the real-world needs of these patients puts them in a position of increased risk of receiving inappropriate interventions. It is thus essential to gain new knowledge into their pathways to care, treatment offered and broader long-term health outcomes. This study aims at overcoming such a gap in knowledge by describing (i) the pathways to care, (ii) the treatment received and (iii) the long-term health outcomes other than risk to develop any persistent psychoses in a large cohort of ATPDs cases.

\section{Methods}

\subsection{Data source}

Data for this study were extracted from the South London and Maudsley (SLaM-BRC) Case Register, using the Clinical Record Interactive Search tool (CRIS) [9]. SLaM is a large NHS Trust providing specialist mental health care to an area of 1.3 million residents across London [9]. Every patient within SLaM has their electronic health record, which is continually updated by SLaM health-care professionals as a legal requirement [9]. CRIS was approved by the Oxfordshire Research Ethics Committee C (reference 08/H0606/71+5). CRIS has been used in over 70 previous studies [10-12]

\subsection{Study population}

Individuals with an index primary diagnosis of ATPDs (F23, ICD10) within SLaM between $1^{\text {st }}$ April 2006 and $15^{\text {th }}$ June 2017 were initially considered eligible [9]. To make the diagnoses more robust, we excluded those who developed a psychotic disorder other than ATPDs within the 3 months immediately following the first index diagnosis (i.e., in the context of the index episode itself) [13].

\subsection{Follow-up}

Follow-up started at the time of the index diagnosis of ATPDs. The end of follow-up was then placed at several a-priori cutoffs ranging from 1 month to 8 years, as indicated below. In the case of time-dependent outcomes (see statistical analysis), censoring occurred on $15^{\text {th }}$ June 2017 or at the time of the event of interest.

\subsection{Study outcomes}

The primary outcome was to describe: (i) the pathways to care, (ii) the treatment received, and (iii) the long-term outcomes of individuals with ATPDs other than risk to develop a persistent psychotic disorder.

\subsubsection{Pathways to care}

Patients' pathways to care within SLaM follows three steps. The first step is acceptance within SLaM. Patients are assigned to a SLaM team that proceeds with their assessment and evaluates if the service is appropriate for their clinical needs. These are the teams that detect the patients - we will refer to these teams as Assessment team. The second step is diagnosis and treatment. These can be operated by the same team that accepted the patients within SLaM, or by another team (if it is thought that the assessment team was not appropriate for patients' clinical needs). We will refer to the team that makes the definitive diagnosis, delivers the treatment and offers clinical follow-up as the Treatment team.

SLaM teams were categorized as follows: Accident and Emergency (Liaison Psychiatry), Child and Adolescent Mental Health, Early Intervention in Psychosis, Forensic Mental Health, Adult Community Mental Health, Older Adults Mental Health, Physical Health (Liaison Psychiatry), Psychosis Community, Substance abuse and CHR-P services. Given that the Early Intervention Services (EIS) are the deputed services for treatment of ATPDs (and CHR-P services for the similar BLIP designation), we considered them as reference treatment services for comparative analysis.

The third step is discharge by the SLaM treatment team. On the basis of the above considerations, we described the pathways to care with three outcome variables:

1) The type of SLaM service that detect (Assessment team), diagnose, offer treatment and clinical follow-up (Treatment team) to patients.

2) Duration of clinical follow-up offered by Treatment teams.

3) The cumulative incidence of discharges by Treatment teams and time to discharge (time-dependent outcome).

\subsubsection{Treatment}

1) Exposure to antipsychotics, antidepressants, or psychotherapy at 1 month, 3 months, 6 months, 1 year, 2 years, 4 years, 6 years, and 8 years.

2) Cumulative incidence of first antipsychotic prescription and time to first antipsychotic prescription (time-dependent outcome).

\subsubsection{Long term health outcomes other than risk to develop persistent} psychoses

1) The percentage of mental health hospitalizations and compulsory hospital admissions (MHA) at 1 month, 3 months, 6 months, 1 year, 2 years, 4 years, 6 years, and 8 years.

2) The percentage of ATPDs that received at least one mental health hospitalization or one compulsory hospital admission (MHA) at 8 years of follow-up.

3) The overall number of days spent in mental health hospitals at 8 years.

Age and ATPDs subtypes might have an impact on pathways to care and long-term health outcomes, respectively. For this reason, 
additional analyses were conducted to provide data on age-based differences in pathways to care and on ATPDs subtypes differences in long-term health outcomes (see Supplementary material eResults)

\subsection{Statistical analysis}

This electronic health record-based cohort study was performed according to the REporting of Studies Conducted using Observational Routinely-collected health Data statement (RECORD)[14] (eTable1).

We described sociodemographic and clinical characteristics of the sample by mean and standard deviation (SD) for continuous variables, and by absolute and relative frequencies for categorical variables.

Pathways to care was measured as: the percentage of ATPDs detected by Assessment teams and treated by Treatment teams; the duration of clinical follow-up measured as mean length of care (days) under a Treatment team; the cumulative incidence of discharges from Treatment teams and time to discharge, estimated with Kaplan-Meier failure analysis and Greenwood 95\% confidence intervals [15]. Supplementary analyses investigated the differential impact of EIS vs other types of SLaM services on these outcomes.

Treatment was measured as the percentage of ATPDs treated with any antipsychotics (yes/no), antidepressants (yes/no), psychotherapy (yes/no) at 1 month, 3 months, 6 months, 1 year, 2 years, 4 years, 6 years, and 8 years. Supplementary descriptive analyses report the detail of the specific antipsychotic medications prescribed at these time points. Treatment was further measured as the cumulative incidence of first antipsychotic prescription and time to first antipsychotic, estimated with Kaplan-Meier failure analysis and Greenwood 95\% confidence intervals [15].

Long term health outcomes other than risk to develop persistent psychoses was measured as the percentage of mental health hospitalizations and compulsory hospital admissions (MHA) at 1 month, 3 months, 6 months, 1 year, 2 years, 4 years, 6 years, 8 years. We also estimated the percentage of ATPDs that received at least one mental health hospitalization and one compulsory hospital admissions (MHA) over 8 years of follow-up. Finally, we reported the overall number of days spent in mental health hospitals over 8 years of follow-up.

Data were analyzed using STATA 13 (STATA Corp., TX, USA).

\section{Results}

\subsection{Sample characteristics}

Between 1st April 2006 and 15th June 2017, a total of 3286 subjects received a first index diagnosis of ATPDs (F23, ICD-10) within the SLaM NHS Trust. After 3 months, 2561 subjects had retained the index ATPDs diagnosis and were therefore included in the current study. The mean age of the sample at the index episode was $33.58 \pm 18.86$ (range: $5-92$ ) and the male to female ratio 1.08; black and white ethnicities were the most represented (36.11\% and $38.21 \%$, respectively). The most frequent ATPDs subtypes were F23.8/F23.9 (Other/Unspecified Acute Transient Psychotic Disorder). See also Table 1 . The mean follow-up time was $6.15 \pm 3.18$ years (range 0-11). In the current manuscript, point estimates of time-dependent outcomes were reported up to 8-years, when at least 133 individuals were still at risk.

\subsection{Pathways to care}

Almost half (47.52\%) of the individuals with an index diagnosis of ATPDs accessing SLaM (Assessment teams) were detected by general mental health psychiatric services (Adult Community Mental Health Services - 36.46\% and Older Adults Mental health $11.06 \%$ ). Only $8.15 \%$ of ATPDs were detected by EIS and none by CHR-P services (see Fig. 1a). Diagnosis, treatment and clinical follow-up within SLaM (Treatment teams) were mostly delivered by general mental health services (54.45\%); only $18.02 \%$ were treated with EIS and no patients were treated by CHR-P services (see Fig. 1b). The low prevalence of EIS in the assessment and treatment phases of ATPDs did not vary across different age-ranges (see Supplementary material -eResults)

Patients treated with EIS were significantly younger (EIS: $25.10 \pm 5.47$ vs Others: $35.94 \pm 14 ; \mathrm{t}=14.04 ; \mathrm{p}<0.01$ ), more frequently males (EIS: $64.30 \%$ vs Others: $48.99 \%, \chi^{2}=29.33$; $\mathrm{p}<0.01$ ), and black (EIS: $46.45 \%$ vs Others: $35.76, \chi^{2}=27.72$; $\mathrm{p}<0.01)$.

The average clinical follow-up duration provided by Treatment teams was $350.40 \pm 589.90$ days. The cumulative incidence of discharge from Treatment teams was $40.94 \%$ at 3 months $(95 \% \mathrm{CI}$ $38.86-43.09 \%$ ), $49.61 \%$ at 6 months (95\%CI $47.48-51.78 \% \%$ ), $60.48 \%$ at 1 year (95\%CI $58.39-62.59 \%), 69.13 \%$ at 2 years (95\%CI $67.12-$ $71.12 \%), 76.92 \%$ at 4 years (95\%CI $75.03-78.76 \%), 80.40 \%$ at 6 years

Table 1

Sociodemographic and clinical characteristics of patients with a first index diagnosis of ICD-10 Acute and Transient Psychotic Disorder (ATPD).

\begin{tabular}{|c|c|c|c|c|}
\hline \multicolumn{5}{|l|}{ Sociodemographic characteristics } \\
\hline & & $N$ & Mean & $S D$ \\
\hline \multirow[t]{2}{*}{ Age } & & 2559 & 33.58 & 13.86 \\
\hline & & $N$ & Count & $\%$ \\
\hline \multirow[t]{3}{*}{ Gender } & & 2561 & & \\
\hline & Males & & 1333 & 52.05 \\
\hline & Females & & 1228 & 47.95 \\
\hline \multirow[t]{6}{*}{ Self- assigned ethnicity } & & 2481 & & \\
\hline & Any white & & 896 & 36.11 \\
\hline & Any black & & 948 & 38.21 \\
\hline & Any asian & & 195 & 7.86 \\
\hline & Mixed & & 57 & 2.30 \\
\hline & Others & & 385 & 15.52 \\
\hline Clinical characteristics & ICD-10 code & $N$ & Count & $\%$ \\
\hline ATPD subtype & & 2561 & & \\
\hline Acute Polymorphic Psychotic Disorder (APPD) & F23.0 & & 116 & 4.53 \\
\hline APPD with symptoms of Schizophrenia & F23.1 & & 131 & 5.12 \\
\hline Acute Schizophrenia-like Psychotic Disorder & $\mathrm{F} 23.2$ & & 366 & 14.29 \\
\hline Other Acute Predominantly Delusional Psychotic Disorder & F23.3 & & 199 & 4.65 \\
\hline Other/Unspecified Acute Transient Psychotic Disorder & F23.8 or F23.9 & & 1641 & 64.08 \\
\hline
\end{tabular}


a. Assessment Teams

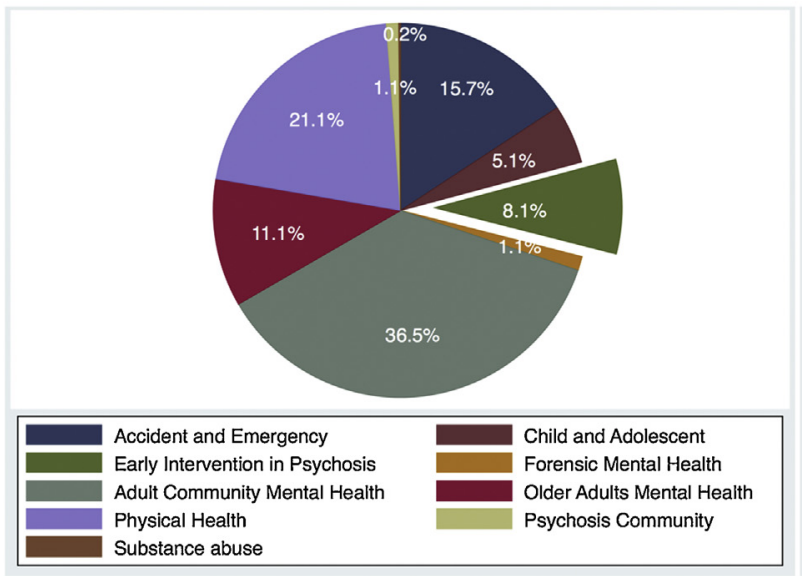

b. Treatment Teams

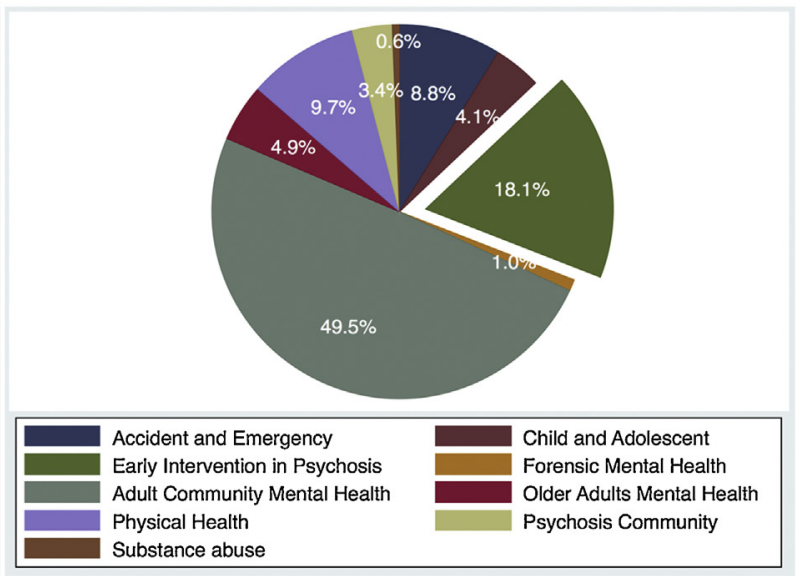

Fig. 1. Pathways to care in patients with Acute and Transient Psychotic Disorders (ATPDs). Percentage of individuals with ATPDs assigned to the different SLaM services for assessment (Fig. 1a) and percentage of individuals with ATPDs assigned to the different SLaM services for diagnosis and treatment (Fig. 1b). No patients were detected/treated by CHR-P services.

(95\%CI $78.55-82.19 \%), 81.59 \%$ at 8 years (95\%CI $79.70-83.39 \%$ ) (see eFig. 1). Supplementary analyses reported in eFig. 2 indicate that patients under the EIS have a longer clinical follow-up and are less likely to be discharged within the 4 years of follow-up.

\subsection{Treatment}

Fig. 2 describes treatments received by ATPDs patients over time. The cumulative incidence of first antipsychotic prescription was $57.77 \%$ at 3 months (95\% CI 55.86-59.69\%); $63.88 \%$ at 6 months (95\% CI 62.02-65.74); 68.95\% at 1 year (95\% CI 67.15-70.74\%); 70.71 at 2 years (95\% CI $68.93-72.48) ; 73.98 \%$ at 4 years (95\% CI $71.61-$ $75.12 \%) ; 74.44 \%$ at 6 years ( $95 \% \mathrm{CI} 72.62-76.18 \%) ; 75.69 \%$ at 8 years (95\% CI 73.86-77.48\%) (see eFig. 3). Time to first antipsychotic medication was $120.55 \pm 327.94$

In eFig. 4 we reported the specific antipsychotic medication prescribed at the time points of interest, indicating that olanzapine is the most frequently prescribed antipsychotic.

\subsection{Long-term health outcomes}

The percentage of ATPDs that received at least one mental health hospitalization and one compulsory mental admission (MHA) over 8 years of follow-up were $32.88 \%$ and $28.54 \%$, respectively (Fig. 3).

The mean duration of mental health hospitalization within SLaM was $66.39 \pm 239.44$ days. Long-term health outcomes did not largely differ between different ATPDs subtypes (see Supplementary material - eResults)

\section{Discussion}

To the best of our knowledge this is the first study reporting on pathways to care, treatment, and long-term health outcomes other than transition to persistent psychotic disorders in a large sample of individuals with ATPDs. The study was conducted using an electronic case register that reflects the day-to-day clinical practice

\section{- ANTIPSYCHOTICS — PSYCHOTHERAPY —ANTIDEPRESSANTS}

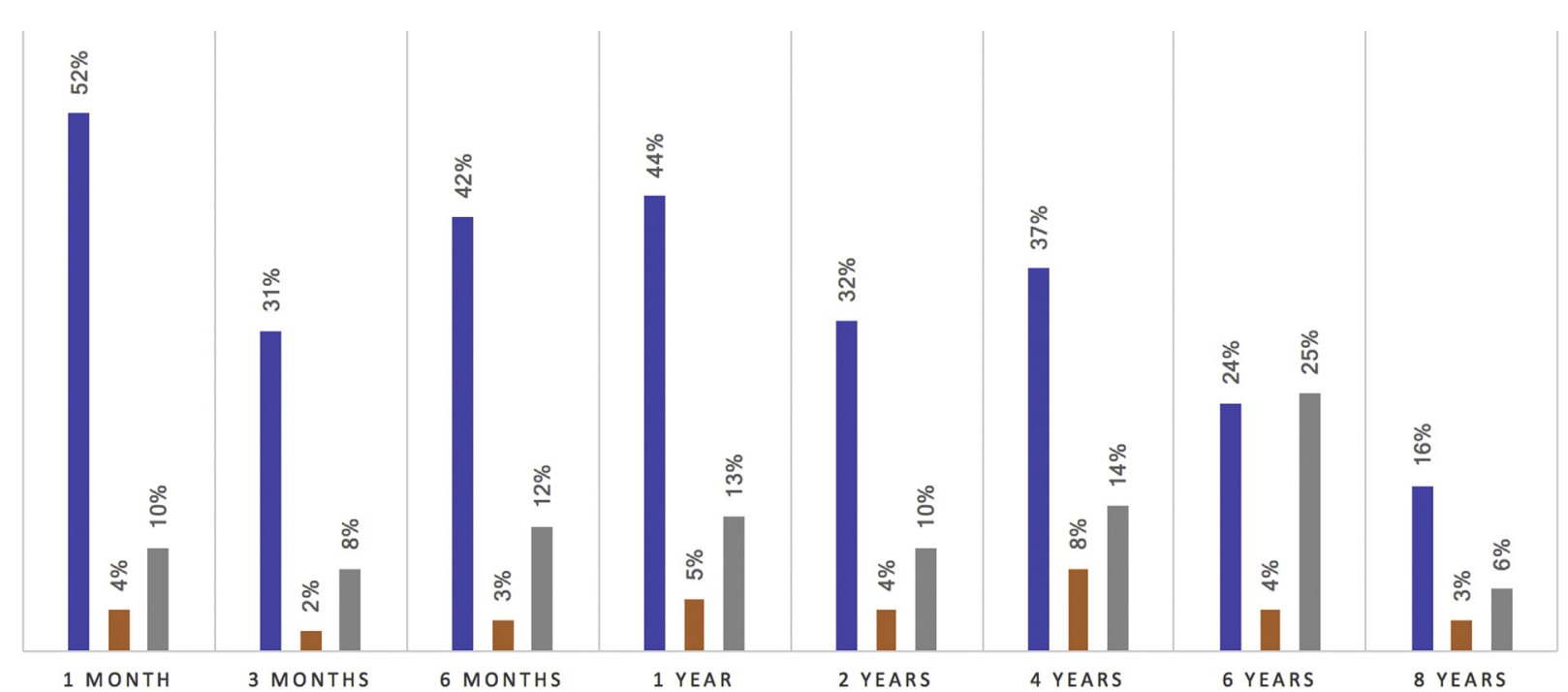

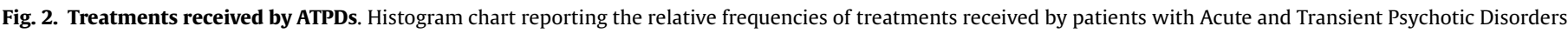
(ATPDs) at different time points. 


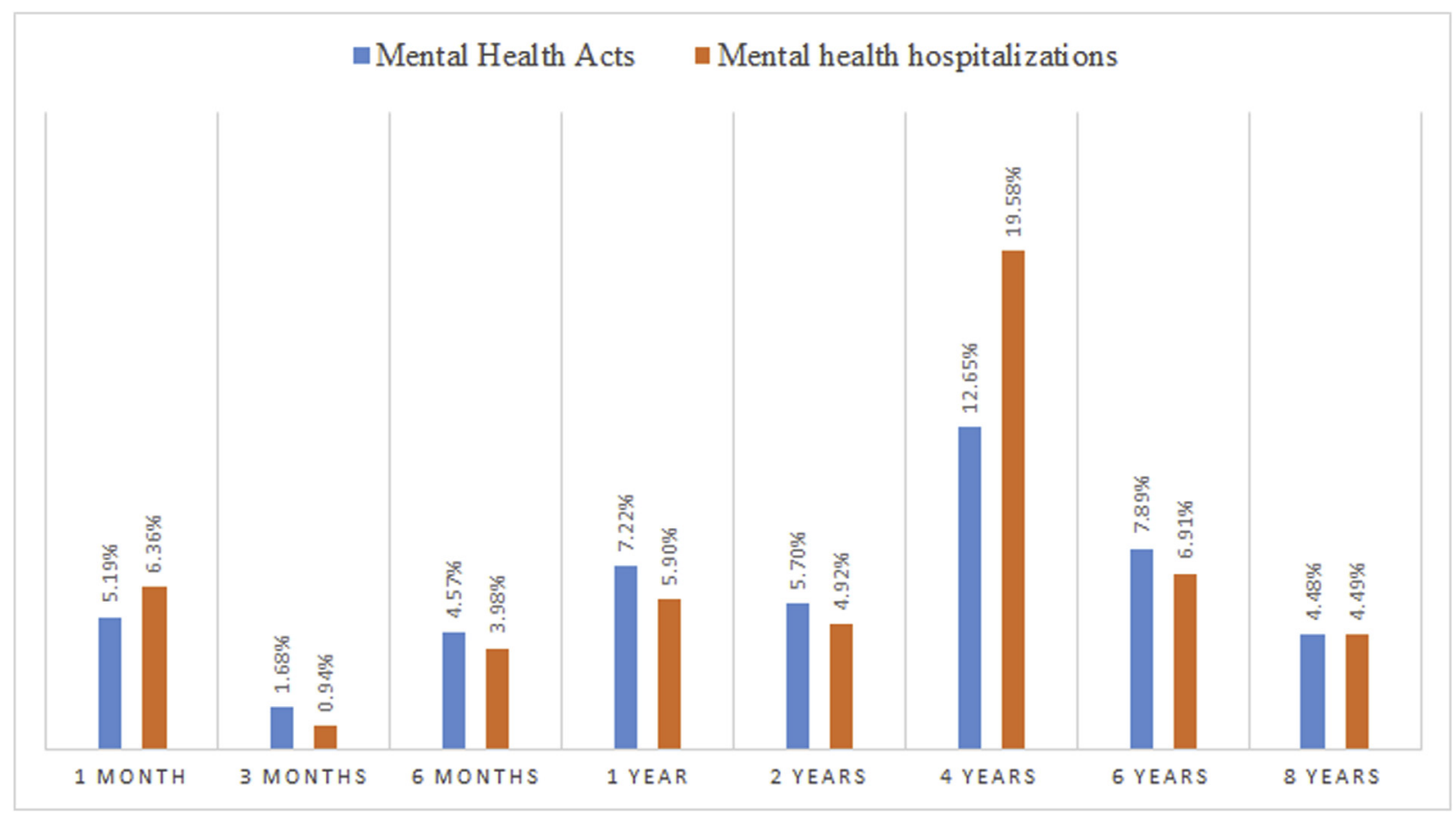

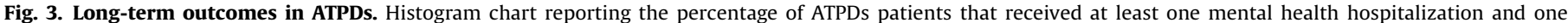
compulsory hospital admission (under the Mental Health Act) over 8 years of follow-up.

of a NHS Mental Health Trust in the UK and, as such, it has high ecological validity. We found that in clinical practice, the majority of ATPDs were not detected or treated by EIS or CHR-P services, received heterogeneous treatments and only short-term clinical follow-up. A substantial proportion of ATPDs would develop severe clinical outcomes beyond persistent psychotic disorders. These patients have unmet clinical needs that are not targeted by current mental health services.

The first outcome of this study was pathway to care for ATPDs patients. Only a minority of patients were detected (8.15\%) and treated $(18.02 \%)$ by EIS, the deputed team for first-episode cases and therefore for taking care of ATPDs. Similarly, there were no ATPDs detected by CHR-P services. It can be argued that since CHR$P$ services use a different designation (i.e. BLIPS) [4] these individuals were not retrieved in the current database. Thus, we checked in the data acquired from the local CHR-P service in the same period and catchment area of the current study (the Outreach and Support In South London) [16], and found that only 49 BLIPS had been detected [16,17]. Assuming that about two-third of BLIPS (i.e. $\mathrm{n}=34$ ) would meet ATPDs criteria [4], the final proportion of ATPDs-like cases detected by CHR-P services is still negligible. Overall, only 241 (207 EIS + 34 CHR-P) individuals with short-lived psychotic episodes have been detected out of the 2610 (2561 ATPDs +49 BLIPS) by the deputed mental health services (EIS or CHR-P), accounting for about $9 \%$ of cases. This is likely due to the fact that most ATPDs are too severe for CHR-P services; in fact, the duration of their episode ( 3 months) can extend that required to meet the BLIPS designation ( 7 days)5. At the same time, referrers in the community may be reluctant to refer ATPDs to EIS because their symptoms are remitted at the time of the diagnosis. Similarly, EIS may not be keen to accept first episode cases that are not severe enough to present with persistent and disabling symptoms that resemble a first episode of schizophrenia spectrum psychoses. Thus, these patients will end up being referred to and treated with non-specialist community mental health services. In line with this speculation, ATPDs patients detected by EIS were typically younger, more frequently males and of black ethnicity, sociodemographic features that characterize non-affective psychoses $[18,19]$. As a result of the detection failure of the deputy teams (EIS and CHR-P), the large majority of ATPDs patients -almost halfwere indeed detected by general psychiatry services. These teams are not specialized in taking care of patients with a first episode of psychosis. Such a suboptimal detection inevitably leads to inefficient treatments. In fact, ATPDs patients received only a very short clinical follow-up: on average one year, with half of them being discharged from the initial teams after six months. Metaanalytical evidence clearly indicates that clinical follow-up in this group should be offered for at least 4 years, due to ATPDs' risk of developing persistent psychotic disorders, which increases from $30 \%$ at 1 year to $54 \%$ at more than 3 years [5]. In line with these arguments, our supplementary analyses found that EIS teams, who have more clinical expertise in taking care of first-episode psychosis, were offering a longer clinical follow-up (about 1 year longer on average) than standard teams. EIS were also less likely to discharge ATPDs patients in the shorter term: within the first three months only a minority (21.15\%) of ATPDs patients were discharged by EIS teams, compared to more than half $(60.11 \%)$ of those treated by other teams (see eFig. 2).

Our second outcome was treatment received by ATPDs patients. In the context of short-term clinical follow-up and early discharge from clinical teams, only $18.02 \%$ of ATPDs were treated by EIS. As such, the type of treatment offered by general mental health teams was not specific and quite heterogeneous, with olanzapine being the most frequently prescribed antipsychotic. Although ATPDs are self-remitting, almost everyone received antipsychotics over the follow-up period and one in ten were prescribed antidepressants. The use of antipsychotics in ATPDs is relatively under-reported, with only a few randomized trials that have investigated conventional antipsychotic treatment, the recommended treatment for a first episode of psychosis [20-22]. The consequent lack of precise therapeutic guidelines might explain why treatment received by ATPDs in the current study was not specific and 
heterogeneous. Interestingly, only a tiny minority of patients received psychotherapy interventions (less than $4 \%$ within the first 3 months). This may both indicate that psychotherapy is not offered by clinicians or accepted by patients, or is not available in the general psychiatry teams where the majority of patients were treated. In the context of CHR-P services, we have observed that BLIPS patients are less likely to accept psychological therapies and have high drop-out rates from these.

As recently highlighted by our research group, one-fourth of patients with an initial ATPDs diagnosis would develop schizophrenia-spectrum psychoses and up to one-third affective psychoses [23]. Our third aim was therefore to investigate longterm outcomes other than risk to develop a persistent psychotic disorder. $32.88 \%$ and $28.54 \%$ of ATPDs received at least one mental health hospitalization or a compulsory hospital admission (MHA) over the 8 years of follow-up, respectively. A substantial proportion considering that this group is traditionally perceived to have a good prognosis and to not require long-term care or follow-up. For those ATPDs who were hospitalised, inpatient admission was not short and lasted on average about 2 months. Considering that half of patients with an ATPDs index diagnosis ultimately developed a persistent psychotic disorder over follow-up [5,24], these results clearly indicate that ATPDs patients are at increased risk of serious long-term outcomes. The high long-term clinical needs of ATPDs contrasts with the relatively short clinical follow-up, high discharge rates and heterogeneous treatments observed in this group, confirming that these patients currently present with unmet needs that are not targeted by existing mental health services.

Improving the care for ATPDs patients starts -as a first stepfrom a more efficient detection by specialized services which can offer them appropriate longitudinal follow-up and care. The BLIPS designation has been introduced specifically to facilitate an early detection of patients with short-lived psychotic episodes and their longitudinal care [25], including prevention of poor long-term outcomes [26]. However, on the ground, the BLIPS approach has failed, because it has demonstrated no pragmatic utility to detect individuals with short-lived psychotic episodes in the real-world clinical scenarios. Clinicians working in the NHS prefer using the ATPDs designation when dealing with short-lived psychotic episodes, and they also do not feel that these patients should be referred to the deputy services who should take care of these patients. The pragmatic failure of the CHR-P paradigm in detecting people at risk of developing psychosis has recently been reported by our group [7,13,27]. Notably, this failure has been observed in the context of well-established CHR-P services with extensive outreach to promote referrals from NHS clinicians. Further investing in outreach campaigns in the local Mental Health Trust to persuade clinicians to refer these patients to the deputy services is unlikely to be successful, because these strategies are already ongoing and demonstrate high inefficiency [7]. Rather, a recent automatized calculator has been developed to screen at scale individuals accessing mental health Trusts and detect those who may be at high risk of developing persistent psychotic disorders [13]. This calculator is particularly useful to detect ATPDs patients. This clinical risk prediction model employs data collected as part of clinical routine in electronic health registries such as the one that has been used to perform the current study. Leveraging on these data, it allows an individualized prediction of outcomes in ATPDs patients, facilitating the treatment or follow-up offered to these patients. The validity of the calculator has recently been replicated in another mental health trust and a pilot study is currently testing the feasibility of using it in the real world mental health care [28]. The second step for improving the care of these patients would be to reconciliate the different operationalizations employed to identify these patients [29], and the inefficient split between EIS and CHR-P services, which is constructed around arbitrary thresholds such as more or less than 7-days duration of the initial episode. The third step would be to refine interventional research for these patients, and to update treatment guidelines accordingly.

The present study has some limitations.

Diagnoses recorded in CRIS as part of clinical routine are excellent in terms of ecological validity, but they are not validated with research-based criteria. However, data recorded in electronic health registers has previously been shown to have good reliability for psychotic disorders [30,31]. Further, in previous studies we have demonstrated that the use of these diagnoses is empirically valid, even outside the local mental health trust [28].

Patients were not systematically assessed during follow-up. We relied on clinical routine data, which is influenced by patient and service-related factors. However, we recently validated our approach, by showing that psychotic outcomes that were estimated through our electronic case register were consistent with those reported by other sites [12].

Finally, we did not report data on future transition to persistent psychotic disorders. It is possible to hypothesize that the long-term outcomes reported in this manuscript overlaps, at least partially, with transition to a persistent psychotic disorder.

\section{Conclusions}

In real-word clinical practice, the majority of ATPDs are not detected or treated by EIS or CHR-P services, receive heterogeneous treatments and only short-term clinical follow-up. ATPDs have a high risk of developing severe clinical outcomes beyond persistent psychotic disorders. These patients have unmet clinical needs that are not targeted by current mental health services.

\section{Financial support}

This work was supported by an MRC grant (MC_PC_16048) to Paolo Fusar-Poli; a Medical Research Council (MRC) Health Data Research UK Fellowship (MR/S003118/1) and a Medical Research Council (MRC) Health Data Research UK Fellowship (MR/S003118/ 1) and a Starter Grant for Clinical Lecturers (SGL015/1020) supported by the Academy of Medical Sciences, The Wellcome Trust, MRC, British Heart Foundation, Arthritis Research UK, the Royal College of Physicians and Diabetes UK to Rashmi Patel.

\section{Conflict of interests}

None.

\section{Acknowledgments}

This work is supported by a King's College (KCL) award (MC_PC_16048) from the Medical Research Council (MRC) to PFP, a MRC Health Data Research UK Fellowship and a Medical Research Council (MRC) Health Data Research UK Fellowship (MR/ S003118/1) and a Starter Grant for Clinical Lecturers (SGL015/1020) supported by the Academy of Medical Sciences, The Wellcome Trust, MRC, British Heart Foundation, Arthritis Research UK, the Royal College of Physicians and Diabetes UK to Rashmi Patel. CRIS is supported by the NIHR-Mental Health, BRC Nucleus at the SLaM NHS Foundation Trust and IoPNN-KCL funded by the Guy's and St Thomas' and the SLaM Trustees.

\section{Appendix A. Supplementary data}

Supplementary material related to this article can be found, in the online version, at doi:https://doi.org/10.1016/j.eurpsy.2018.12.006. 


\section{References}

[1] Biedermann F, Fleischhacker WW. Psychotic disorders in DSM-5 and ICD-11. CNS Spectr 2016;21(04):349-54, doi:http://dx.doi.org/10.1017/ S1092852916000316.

[2] Fusar-Poli P, Cappucciati M, Borgwardt S, Woods SW, Addington J, Nelson B, et al. Heterogeneity of psychosis risk within individuals at clinical high risk. JAMA Psychiatry 2016;73(February (2)):113-20, doi:http://dx.doi.org/10.1001/ jamapsychiatry.2015.2324.

[3] Fusar-Poli P. The clinical high-risk state for psychosis (CHR-P), version II. Schizophr Bull 2017;43(1):44-7, doi:http://dx.doi.org/10.1093/schbul/sbw158.

[4] Fusar-Poli P, Cappucciati M, De Micheli A, Rutigliano G, Bonoldi I, Tognin S, et al. Diagnostic and prognostic significance of brief limited intermittent psychotic symptoms (BLIPS) in individuals at ultra high risk. Schizophr Bull 2017;43(1):48-56, doi:http://dx.doi.org/10.1093/schbul/sbw151.

[5] Fusar-Poli P, Cappucciati M, Bonoldi I, Hui LM, Rutigliano G, Stahl DR, et al. Prognosis of brief psychotic episodes: a meta-analysis. JAMA Psychiatry 2016;73(3):211-20, doi:http://dx.doi.org/10.1001/jamapsychiatry.2015.2313.

[6] Fusar-Poli P, Cappucciati M, Rutigliano G, Heslin M, Stahl D, Brittenden Z, et al. Diagnostic stability of ICD/DSM first episode psychosis diagnoses: metaanalysis. Schizophr Bull 2016;42(6):1395-406, doi:http://dx.doi.org/10.1093/ schbul/sbw020.

[7] Fusar-Poli P. Extending the benefits of indicated prevention to improve outcomes of first-episode psychosis. JAMA Psychiatry 2017;74(7):667, doi: http://dx.doi.org/10.1001/jamapsychiatry.2017.1009.

[8] NICE. Psychosis and schizophrenia in adults: prevention and management | Guidance and guidelines. NICE; 2018. . (Accessed May 17, 2018) https://www. nice.org.uk/Guidance/CG178.

[9] Stewart R, Soremekun M, Perera G, Broadbent M, Callard F, Denis M, et al. The South London and Maudsley NHS Foundation Trust Biomedical Research Centre (SLAM BRC) case register: development and descriptive data. BMC Psychiatry 2009;9(August 12):51, doi:http://dx.doi.org/10.1186/1471-244X-9-51.

[10] Perera G, Broadbent M, Callard F, Chang CK, Downs J, Dutta R, et al. Cohort profile of the South London and Maudsley NHS Foundation Trust Biomedical Research Centre (SLaM BRC) Case Register: current status and recent enhancement of an Electronic Mental Health Record-derived data resource. BMJ Open 2016;6(3) e008721, doi:http://dx.doi.org/10.1136/bmjopen-2015-008721.

[11] Roberts E, Wessely S, Chalder T, Chang C-K, Hotopf M. Mortality of people with chronic fatigue syndrome: a retrospective cohort study in England and Wales from the South London and Maudsley NHS Foundation Trust Biomedical Research Centre (SLaM BRC) Clinical Record Interactive Search (CRIS) Register. Lancet (London, England) 2016;387(10028):1638-43, doi:http://dx.doi.org/ 10.1016/S0140-6736(15)01223-4.

[12] Fusar-Poli P, Rutigliano G, Stahl D, Schmidt A, Ramella-Cravaro V, Hitesh S, et al. Deconstructing pretest risk enrichment to optimize prediction of psychosis in individuals at clinical high risk. JAMA Psychiatry 2016;73 (12):1260, doi:http://dx.doi.org/10.1001/jamapsychiatry.2016.2707.

[13] Fusar-Poli P, Rutigliano G, Stahl D, Davies C, Bonoldi I, Reilly T, et al Development and validation of a clinically based risk calculator for the transdiagnostic prediction of psychosis. JAMA Psychiatry 2017;74(5):493, doi: http://dx.doi.org/10.1001/jamapsychiatry.2017.0284.

[14] Benchimol EI, Smeeth L, Guttmann A, Harron K, Moher D, Petersen I, et al. The reporting of studies conducted using observational routinely-collected health data (RECORD) statement. PLOS Med 2015;12(10)e1001885, doi:http://dx.doi. org/10.1371/journal.pmed.1001885.

[15] Farewell V, Johnson T. Major Greenwood (1880-1949): a biographical and bibliographical study. Stat Med 2016;35(5):645-70, doi:http://dx.doi.org/ 10.1002/sim.6772.
[16] Fusar-Poli P, Byrne M, Badger S, Valmaggia LR, McGuire PK. Outreach and support in South London (OASIS), 2001-2011: ten years of early diagnosis and treatment for young individuals at high clinical risk for psychosis. Eur Psychiatry 2013;28(5):315-26, doi:http://dx.doi.org/10.1016/j. eurpsy.2012.08.002.

[17] Fusar-Poli P, Rutigliano G, Stahl D, Davies C, De Micheli A, Ramella-Cravaro V, et al. Long-term validity of the At Risk Mental State (ARMS) for predicting psychotic and non-psychotic mental disorders. Eur Psychiatry 2017;42:49-54, doi:http://dx.doi.org/10.1016/j.eurpsy.2016.11.010.

[18] Millan MJ, Andrieux A, Bartzokis G, Cadenhead K, Dazzan P, Fusar-Poli P, et al. Altering the course of schizophrenia: progress and perspectives. Nat Rev Drug Discov 2016;15(7):485-515, doi:http://dx.doi.org/10.1038/nrd.2016.28.

[19] Correll CU, Penzner JB, Frederickson AM, et al. Differentiation in the preonset phases of schizophrenia and mood disorders: evidence in support of a bipolar mania prodrome. Schizophr Bull 2007, doi:http://dx.doi.org/10.1093/schbul/ sbm028.

[20] Agarwal V, Sitholey P. A preliminary open trial of olanzapine in paediatric acute and transient psychotic disorders. Indian J Psychiatry 2006;48(1):43-6, doi:http://dx.doi.org/10.4103/0019-5545.31618.

[21] Chaudhuri BP, Bhagabati D, Medhi D. Risperidone versus haloperidol in acute and transient psychotic disorder. Indian J Psychiatry 2000;42(3)280-90. . (Accessed April 18, 2018) http://www.ncbi.nlm.nih.gov/pubmed/21407958.

[22] Khanna A, Lal N, Dalal PK, Khalid A, Trivedi JK. Treatment of acute and transient psychotic disorders with low and high doses of oral haloperidol. Indian J Psychiatry 1997;39(2)136-42. . (Accessed April 18, 2018) http://www.ncbi. nlm.nih.gov/pubmed/21584060.

[23] Rutigliano G, Merlino S, Minichino A, Patel R, Davies C, Oliver D, et al. Long term outcomes of acute and transient psychotic disorders: the missed opportunity of preventive interventions. Eur Psychiatry 2018;52, doi:http:// dx.doi.org/10.1016/j.eurpsy.2018.05.004.

[24] Fusar-Poli P, Cappucciati M, Bonoldi I, Hui LM, Rutigliano G, Stahl DR, et al Prognosis of brief psychotic episodes. JAMA Psychiatry 2016;73(3):211, doi: http://dx.doi.org/10.1001/jamapsychiatry.2015.2313.

[25] McGorry P, Nelson B. Why we need a transdiagnostic staging approach to emerging psychopathology, early diagnosis, and treatment. JAMA Psychiatry 2016;73(3):191, doi:http://dx.doi.org/10.1001/jamapsychiatry.2015.2868.

[26] Fusar-Poli P, McGorry PD, Kane JM. Improving outcomes of first-episode psychosis: an overview. World Psychiatry 2017;16(3):251-65, doi:http://dx. doi.org/10.1002/wps.20446.

[27] Fusar-Poli P. The hype cycle of the clinical high risk State for psychosis: the need of a refined approach. Schizophr Bull 2018;44(2):250-3, doi:http://dx. doi.org/10.1093/schbul/sbx181.

[28] Fusar-Poli P, Werbeloff N, Rutigliano G, Oliver D, Davies C, Stahl D, et al Transdiagnostic risk calculator for the automatic detection of individuals at risk and the prediction of psychosis: second replication in an independent National Health Service Trust. Schizophr Bull 2018(June), doi:http://dx.doi. org/10.1093/schbul/sby070.

[29] Fusar-Poli P, Cappucciati M, Bonoldi I, et al. Prognosis of brief psychotic episodes. JAMA Psychiatry 2016;73(3):211, doi:http://dx.doi.org/10.1001/ jamapsychiatry.2015.2313.

[30] Davis KAS, Sudlow CLM, Hotopf M. Can mental health diagnoses in administrative data be used for research? A systematic review of the accuracy of routinely collected diagnoses. BMC Psychiatry 2016;16(1):263, doi:http://dx.doi.org/10.1186/s12888-016-0963-x.

[31] Pihlajamaa J, Suvisaari J, Henriksson M, Heilä H, Karjalainen E, Koskela J, et al. The validity of schizophrenia diagnosis in the Finnish Hospital discharge register: findings from a 10-year birth cohort sample. Nord J Psychiatry 2008;62(3):198-203, doi:http://dx.doi.org/10.1080/08039480801983596. 